\title{
Salivary parameters among chronic alcoholics and non-alcoholic males in Ambala, Haryana - a comparative study
}

\begin{abstract}
Background: Salivary diagnostics is a dynamic and emerging field utilizing molecular diagnostics to aid in the diagnosis of oral and systemic diseases. The present study was conducted to assess salivary parameters (flow rate, ph and buffer capacity) among alcoholics and non-alcoholics.

Materials and Method: A total number of 100 male subjects (50 cases and 50 controls) between the age of 18 years to 49 years were examined. Various salivary parameters like $\mathrm{pH}$, buffering capacity and salivary flow rate in chronic alcoholic and non- alcoholic male subjects were assessed. Data was analyzed by SPSS package version 13.p-value $\leq 0.05$ and $\leq 0.01$ was considered as significant and highly significant respectively.
\end{abstract}

Results: The difference between the salivary flow rates among alcoholics and nonalcoholics were highly significant $(\mathrm{p}<0.001)$. The salivary $\mathrm{pH}$ was more acidic in alcoholics, only $10 \%$ of the alcoholics compared to $62 \%$ of the non-alcoholics had normal salivary $\mathrm{pH} .24 \%$ (12) of alcoholic subjects had normal salivary buffer capacity as compared to $94 \%$ (47) of non-alcoholic subjects who had normal salivary buffer capacity. The difference among two groups was highly significant.

Conclusion: Salivary diagnostics serves as a subset of the larger field of molecular diagnostics, now recognized as a central player in a wide variety of biomedical basic and clinical areas. Present study shows a significant relationship between smoking and decreased parameters (having anti caries effect) salivary flow and ph.

Keywords: saliva, alcohol, buffer-capacity, pH, flowrate
Volume 9 Issue 5 - 2018

Tarun Gupta,' KL Veeresha, ${ }^{2}$ Tanvir Singh Teja, ${ }^{3}$ Dilpreet Kaur, ${ }^{4}$ Ramandeep Singh Gambhir $^{5}$

'Department of Public Health Dentistry, Lakshmi Bai Dental College and Hospital, India

${ }^{2}$ Department of Public Health Dentistry, MM College of Dental Sciences and Research, India

${ }^{3}$ Department of Endodontics and Conservative Dentistry, Rayat and Bahra Dental College and Hospital, India

${ }^{4}$ Department of General Dentistry, Dental Aesthetix Clinic, India ${ }^{5}$ Department of Public Health Dentistry, BRS Dental College and Hospital, India

Correspondence: Ramandeep Singh Gambhir, Reader and Head, BRS Dental College and Hospital, Panchkula, Pin I34 I I8, India, Tel +919915646007, Email raman2g@yahoo.com

Received: September 05, 2018 | Published: October 09, 2018

\section{Introduction}

Alcohol dependency is the most neglected public health problem in today's world. ${ }^{1}$ According to the World Health Organization (2002), alcohol use is the fifth leading risk factor for premature death around the world, following low-weight (malnourished), unsafe sex, high blood pressure, and tobacco. Alcohol is the most widely used mood altering liquid substance. It serves a vast range of religious, culinary, psychic, ceremonial, hedonistic, traditional, social and medicinal purpose in the modern society. It is unlikely that a person begins to drink with the intention of becoming an alcoholic. Alcohol use is also strongly leads to social consequences such as drink driving injuries and fatalities, aggressive behaviour, family disruptions and reduced industrial productivity. ${ }^{2,3}$ Alcoholism may imply social consequences such as unemployment or early retirement, as well as toxic effects on general health such as liver cirrhosis. Alcoholics frequently show overt signs of poor oral hygiene including carious teeth, calculus and coated tongue. ${ }^{2}$ There is also an increased prevalence of generalized periodontitis with tooth loss among alcoholics as compared to nonalcoholics. ${ }^{1-5}$ Alcohol is consistently reported as being related with increased rates of oral mucosal lesions, head and neck cancer and a high percentage of tooth wear in the form of attrition and erosion leading to decrease in quality of life. ${ }^{4,6}$ In addition, the promotion, popularity, range and availability of inexpensive alcoholic beverages have increased. These developments have likely contributed to adolescents initiating heavier drinking at younger ages than ever before.

Alcohol related problems are greatly increasing and becoming so common among the general population that they are making a significant impact on the day to day practice of medicine and dentistry. ${ }^{6}$ An extensive alcohol consumption may affect oral health in several ways: in the first place, a direct effect, for example, in case of caries due to alcohol-related xerostomia; secondly, an indirect effect ascribed to changes in dental health behaviour. ${ }^{5}$ Alcoholism is a condition which alters salivary flow rates and composition diminishing the production and quality of saliva. However, there are not many studies reported to assess salivary parameters and oral disease among alcoholics. Hence this study was taken up to assess salivary parameters (flow rate, ph and buffer capacity) among alcoholics and non-alcoholics.

\section{Material and methods}

\section{Ethics and informed consent}

The study was conducted among patients visiting the outpatient Department (OPD) of Public Health Dentistry, M.M college of Medical Sciences and Research, Mullana to correlate the salivary parameters in male alcoholics and non- alcoholics between 18-49 years of age in Ambala District Haryana and to calibrate salivary parameters- $\mathrm{pH}$, buffering capacity and salivary flow rate in chronic alcoholic and non- alcoholic male subjects. Ethical approval from the institutional ethical committee (IEC) was taken and written informed consent was obtained from the study subjects prior to the study.

\section{Study sample}

The study population comprised of only male subjects who were vising the Out-patient Department (OPD) of Public Health Dentistry 
for routine check-up and treatment. The sample size for the present study was determined on the basis of a pilot study conducted on 20 subjects. After doing all the calculations, a total of 100 male subjects were engaged in the present study. Female subjects were not included in the study as alcohol consumption among females is considered as taboo and culturally not acceptable in a state like Haryana. On the basis of simple random sampling, subjects were equally divided into two groups-cases and controls.

\section{Inclusion and Exclusion criteria}

The Inclusion criteria for case group were 50 male subjects who daily consume more than three drinks or more than 14 drinks in one week (one drink equivalent to $30 \mathrm{ml}$ of alcohol) for past 6 months and were between 18-49 years of age. Exclusion criteria for case group were alcoholic subjects with other habits like smoking and chewing tobacco, aged 50 and above, with systemic disease (confounding factors), with less than six months' alcohol consumption. Inclusion criteria for control group were all males below 50 years of age without alcoholic habit or tobacco habit. Exclusion criteria for control group were subjects with any underlying systemic diseases (confounding factors), aged 50 and above.

\section{Examiner calibration}

The examiner was calibrated by the departmental staff before the study. The case and control groups were selected from the outpatient as per the given criteria of the study. Type III type of examination was conducted utilizing dental unit with illuminating light and proper armamentarium. The recording was done by a trained intern who was made to sit close to the examiner. Demographic details, details of deleterious habits and oral experiences were entered on a structured format. Certain general conditions like anaemia and BMI were recorded for referral purpose. Salivary Assessment was done by using GC Saliva Check Buffer Kit. Salivary parameters were assessed before oral examination.

\section{Collection of Saliva}

Saliva samples were collected from all subjects between 9-11 am to avoid circadian variations. The whole saliva was collected by spitting method. Before collection of saliva the mouth was emptied by an initial swallow. The unstimulated (resting) whole saliva was collected by asking the patient to allow the saliva to pool in the mouth for five minutes and then spit into a graduated cup. After clearing the mouth by swallowing accumulated saliva, each subject was asked to chew on a strip of paraffin wax and asked to expectorate at 60 seconds intervals for five minutes. The collected saliva was quantified as $\mathrm{mL} /$ min of saliva. Thus a flow rate of saliva was assessed. The $\mathrm{pH}$ of the unstimulated and stimulated saliva was estimated by dipping the $\mathrm{pH}$ test paper directly into the sample of saliva to wet it and then removing it immediately. The colour change can be matched directly with the scale provided by the manufacturer and individual $\mathrm{pH}$ values were recorded. The assessment of buffering capacity of the stimulated saliva was done using buffer test strips provided in the kit. The grading of the buffering capacity was done according to the colour changes and scores obtained as specified by the manufacturer. All the calibrated salivary parameters were entered on the structured format.

\section{Statistical analysis}

Data was analyzed by SPSS package version 13. Student t-test was used for statistical analysis. P-value $\leq 0.05$ and $\leq 0.01$ was considered as significant and highly significant respectively.

\section{Results}

\section{Salivary parameters}

Study group: Among 50 alcoholic subjects 52\% (26) were having low salivary flow rate, $32 \%$ (16) were having very low salivary flow rate and only $16 \%(8)$ had normal salivary flow rate.

Control group: Among 50 non-alcoholic subjects no subject had very low salivary flow rate, $22 \%$ (11) were having low salivary flow rate and $78 \%$ (39) had normal salivary flow rate. $\mathrm{P}$-value is $<.001$ which is highly statistically significant (Table 1) (Figure 1).

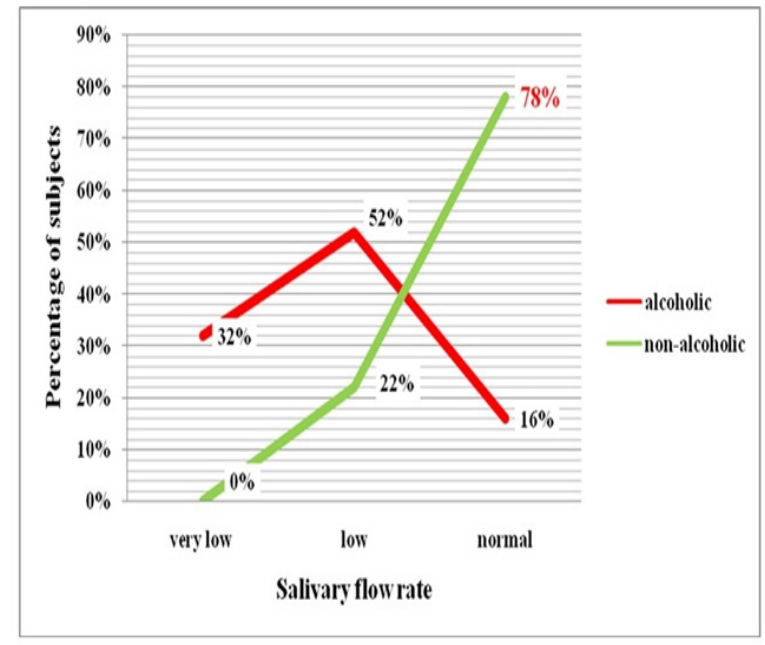

Figure I Showing distribution of subjects according to their salivary flow rates.

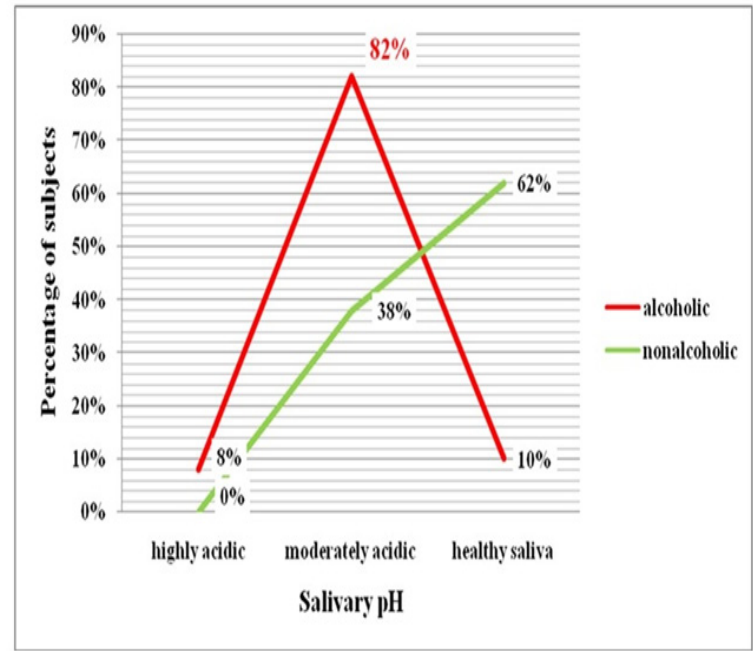

Figure 2 Showing distribution of subjects according to their salivary $\mathrm{pH}$. 
Table I Showing distribution of subjects according to various salivary parameters

**Highly statistically significant

\begin{tabular}{|c|c|c|c|c|c|c|}
\hline \multirow{2}{*}{ Salivary parameters } & \multirow{2}{*}{ Readings } & \multicolumn{2}{|c|}{ Alcoholic } & \multicolumn{2}{|c|}{ Non-Alcoholic } & \multirow{2}{*}{ p-value } \\
\hline & & $\mathbf{N}$ & \%age & $\mathbf{N}$ & \%age & \\
\hline \multirow{3}{*}{ Flow Rate } & $<3.5 \mathrm{~mL}$ - very low & 16 & $32 \%$ & 0 & $0 \%$ & \multirow{3}{*}{$<.001 * *$} \\
\hline & $5.0-3.5 \mathrm{~mL}-$ low & 26 & $52 \%$ & II & $22 \%$ & \\
\hline & $>5.0 \mathrm{~mL}-$ Normal & 8 & $16 \%$ & 39 & $78 \%$ & \\
\hline \multirow{3}{*}{$\mathrm{pH}$} & 5- 5.8 highly acidic & 4 & $8 \%$ & 0 & $0 \%$ & \multirow{3}{*}{$<.001 * *$} \\
\hline & 6.0-6.6 moderately acidic & 41 & $82 \%$ & 19 & $38 \%$ & \\
\hline & 6.8-7.8 normal pH & 5 & $10 \%$ & 31 & $62 \%$ & \\
\hline \multirow{3}{*}{ Buffer Capacity } & $0-5$ very low & 2 & $4 \%$ & 0 & 0 & \multirow{3}{*}{$<.001 * *$} \\
\hline & 6-9 low & 36 & $72 \%$ & 3 & $6 \%$ & \\
\hline & $10-12$ normal & 12 & $24 \%$ & 47 & $94 \%$ & \\
\hline
\end{tabular}

\section{II-Salivary pH}

problem. Habits like smoking and alcohol drinking among females is considered as a strong social taboo in the Indian society. On the other hand it is considered as symbol of social prestige among the males hence alcoholism is one of the most common habit reported in them. India has been identified as the third largest market for alcoholic beverages in the world and state of Haryana is also one of the major contributors. $^{7}$

In the present study more than half of the alcoholics were $<45$ years of age followed by $42 \%$ alcoholics in $35-44$ years age group. This may be due to chronicity of drinking and easy availability of alcohol, strain and stress due to job insecurity, unemployment, family responsibilities and peer pressure. ${ }^{8-10}$ The difference between the salivary flow rates among alcoholics and non-alcoholics were highly significant $(\mathrm{p}<0.001)$. The present study clearly indicates that the salivary $\mathrm{pH}$, flow rate and buffer capacity are significantly lowered in alcoholic patients.

The salivary flow rate in this study was significantly low in alcoholics. Only $16 \%$ of the alcoholics compared to $78 \%$ of the non-alcoholics had normal salivary flow rate. $32 \%$ of alcoholics had marked hypo salivation. In this study mean number of decayed teeth, missing teeth as result of caries increased with decrease in salivary flow rate which may be because reduced salivary flow rate with concomitant reduction of oral defense system may cause severe caries and mucosal inflammation. ${ }^{11,12}$ Dental caries is probably the most common consequence of hyposalivation. ${ }^{13,14}$ Caries lesions develop rapidly and also on tooth surfaces that are usually not susceptible to caries. Subjects with impaired saliva flow rate often show high caries incidence. ${ }^{15-17}$ In this present study mean number missing teeth due any other reason were more when salivary flow rate is low because of poor oral hygiene with concomitant periodontal disease, vitamin deficiencies, and alcohol induced medical illness. One surface filing and two surface filing requirements was more among alcoholics with low salivary $\mathrm{pH}$.

In our present study the salivary $\mathrm{pH}$ was more acidic in alcoholics. Only $10 \%$ of the alcoholics compared to $62 \%$ of the non-alcoholics had normal salivary $\mathrm{pH}$. It has been observed that patients with alcoholism maybe at a greater risk of developing dental lesions owing to low $\mathrm{pH}$ level of their oral environment and decreased salivary levels. ${ }^{18}$ Some other authors also reported similar findings and concluded that lowered $\mathrm{pH}$ of saliva ( $\mathrm{pH}$ between 3-3.8) may be attributed to consumption of alcohol. ${ }^{19}$ Alcoholics with low salivary $\mathrm{pH}$ required more than one surface filing. Mean number of sound teeth were more in non- alcoholics who were having normal salivary

Figure 3 Showing distribution of subjects according to their salivary buffer capacity.

\section{Discussion}

Alcoholism is a worldwide social and medical public health 
$\mathrm{pH}$ (6.8-7.8) where as more number of decayed teeth, filled teeth with decay, missing teeth due to caries and missing teeth because of other reason were more in alcoholics who were having acidic salivary $\mathrm{pH}$. The presence of low $\mathrm{pH}$ is conducive for non-Mutans streptococci, Lactobacilli bacteria and the normally non-pathogenic Actinomyces sup. Bacteria can adapt to produce acid that destabilizes the homeostatic biofilm causing a shift to a more overall acidogenic plaque biofilm. Mutans streptococci or Lactobacilli bacteria can then predominate at a lower $\mathrm{pH}$ and cause dental caries. ${ }^{20}$

In the present study the salivary buffer capacity was low in alcoholics with only $24 \%$ of the alcoholics compared to $94 \%$ of the non-alcoholics having normal buffering capacity. One surface filing with more mean number of decayed teeth, missing due to dental caries was more among alcoholics who were having low salivary buffer capacity. A low flow rate combined with a low or moderate buffer effect clearly indicates poor salivary resistance against microbial attack. ${ }^{21}$ Higher the flow rate, the faster the oral clearance and the higher the buffer capacity. ${ }^{22}$ The buffer effect of saliva is also affected by hormonal and metabolic changes, as well as by altered general health. ${ }^{23,24}$

\section{Conclusion and recommendations}

Present study shows a significant relationship between alcohol consumption and decreased parameters (having anti caries effect) salivary flow and ph. Hence following recommendations being done:

1) As a primordial care a rapid health education should be taken up by the professionals and NGO's (Non-Government Organizations) utilizing all the available Mass Medias to prevent population from alcohol habit.

2) There should be a strong health education procedures implemented in schools against alcohol addiction which should again made as a part of syllabus so as to prevent younger generation from alcohol addiction.

3) Alcoholics can be considered as one of the risk group for various dental conditions, hence health education should be focused on them and proper tooth brushing and flossing methods should be taught to prevent plaque formation.

As a therapeutic measure, alcoholics with sign of xerostomia may be prescribed artificial salivary products and all the type of interventions and dental treatment can be rendered to the alcoholics on priority basis.

\section{Acknowledgement}

None.

\section{Conflict of interest}

The author declares that there is no conflict of interest.

\section{References}

1. Sullivan JF. Zinc Metabolism and Chronic Alcoholism. Am J Clin Nutr August. 1965;17(2):57-63.

2. Maier H, Born IA, Mall G. Effect of chronic ethanol and nicotine consumption on the function and morphology of the salivary glands. Klin Wochenschr. 1988;66 Suppl 11:140-150.

3. Dutta SK, Dukehart M, Narang A, et al. Functional and structural changes in parotid glands of alcoholic cirrhotic patients. Gastroenterology. 1989;96(2 Pt 1):510-518.
4. Dutta SK, Orestes M, Vengulekur S. Ethanol and human saliva: effect of chronic alcoholism on flow rate, composition, and epidermal growth factor. Am J Gastroenterol. 1992;87(3):350-354.

5. Katharine a. Bradley KA. Management of Alcoholism in the Primary Care Setting. West J Med. 1992;156(3):273-277.

6. Novacek G, Plachetzky U, Pötzi R, et al. Dental and periodontal disease in patients with cirrhosis - role of etiology of liver disease. J Hepatol. 1995;22(5):576-582.

7. Kumar K, Kumar S, Singh AK. Prevalence and socio-demographic correlates of alcohol consumption: Survey findings from five states in India. Drug Alcohol Depend. 2018;185:381-390.

8. Moura EC, Malta DC. Alcoholic beverage consumption among adults: sociodemographic characteristics and trends. Rev Bras Epidemiol. 2011;14 Suppl:61-70.

9. Goswami A, Reddaiah VP, Kapoor SK, et al. Tobacco and alcohol use in rural elderly Indian population. Indian J Psychiatry. 2005;47(4):92-97.

10. Gupta R, Sharma S, Gupta VP, et al. Smoking and alcohol intake in a rural Indian population and correlation with hypertension and coronary heart disease prevalence. J Assoc Physicians India. 1995;43(4):253-258.

11. Daniels TE, Silverman S, Michalski JP, et al. The oral component of Sjogren's syndrome. Oral Surg. 1975;39(6):875-885.

12. Van der Reijden WA, van der Kwaak JS, Veerman ECI, et al. Analysis of the concentration and output of whole salivary constituents in patients with Sjogren's syndrome. Eur J Oral Sci. 1996;104(4 (Pt 1):335-340.

13. Brown LR, Dreizen S, Daly TE, et al. Interrelations of oral microorganisms, immunoglobulins, and dental caries following radiotherapy. J Dent Res. 1978;57(9-10):882-893

14. Scully C. Sjogren's syndrome: clinical and laboratory features, immunopathogenesis, and management. Oral Surg Oral Med Oral Pathol. 1986;62(5):510-523.

15. Gao X, Jiang S, Koh D, et al. Salivary biomarkers for dental caries. Periodontol 2000. 2016;70(1):128-141.

16. Flink H. Studies on the prevalence of reduced salivary flow rate in relation to general health and dental caries, and effect of iron supplementation. Swed Dent J. 2007;(192):3-50.

17. Jawed M, Shahid SM, Qader SA, Azhar A. Dental caries in diabetes mellitus: role of salivary flow rate and minerals. J Diabetes Complications. 2011;25(3):183-186

18. Dukić W, Dobrijević TT, Katunarić M, et al. Erosive lesions in patients with alcoholism. J Am Dent Assoc. 2010;141(12):1452-1458.

19. Chehal HK, Pate DH, Cohen DM, et al. Dental erosion due to excessive wine consumption. Gen Dent. 2009;57(5):519-523.

20. Hurlbutt M, Young DA. A best practices approach to caries management. $J$ Evid Based Dent Pract. 2014;14 Suppl:77-86.

21. Lagerlöf F, Oliveby A. Caries-protective factors in saliva. Adv Dent Res. 1994;8(2):229-238.

22. Cunha-Cruz J, Scott J, Rothen M, et al. Salivary characteristics and dental caries: evidence from general dental practices. J Am Dent Assoc. 2013;144(5):e31-40

23. Siudikiene J, Machiulskiene V, Nyvad B, et al. Dental caries and salivary status in children with type 1 diabetes mellitus, related to the metabolic control of the disease. Eur J Oral Sci. 2006;114(1):8-14.

24. Preoteasa E, Tâncu AM, Iosif L, et al. Salivary changes related to systemic diseases in the edentulous patients. $J$ Med Life. 2014;7(4):577-580. 